\title{
A New Handheld Biochip-based Microsystem
}

\author{
P. A. C. Lopes, J. Germano, \\ T. M. Almeida, L. Sousa and M. S. Piedade \\ INESC-ID / IST \\ Rua Alves Redol n. 9, 1000-029 Lisboa \\ email: paulo.c.lopes@inesc-id.pt
}

\author{
F. Cardoso, H. A. Ferreira \\ P. P. Freitas \\ INESC-NM / IST \\ Rua Alves Redol n. 9, 1000-029 Lisboa \\ email: fcardoso@inesc-mn.pt
}

\begin{abstract}
This paper presents a recently developed handheld biochip-based microsystem. The microsystem is based on a magneto-resistive array biochip composed of a number of sensing sites with magnetic tunneling junctions (MTJ) and diodes. To drive the MTJ, different techniques are addressed with different types of signals. Filtering strategies are also presented, which allow the recovery of bio signals from the noise without increasing too much nor the time required to access all the sensors, nor the power consumption of the board. In conclusion, experiments with the system in a setup to detect actual bio signals are presented with encouraging results.
\end{abstract}

\section{INTRODUCTION}

One of the trends of the last decade has been the miniaturization of typical large laboratories experiments. This was made possible by the advance in microfluids and MicroElectro-Mechanical Systems (MEMS) technologies. One of the outcomes of this trend has been the so called "lab on a chip" systems [1]. In these systems complex chambers and pipes are built directly into a chip using the standard silicon processing technology of integrated circuits. Usually the systems then relies on micro-arrays [2] to convert the results of the experiment into signals to be analyzed in a computer. Microsystems as the one described in this paper offer great promise.

The microsystem described in this paper [3], is based on magnetoresistive biochips. These chips have been introduced for fully integrated biomolecular recognition assays [4], [5]. In these experiments, target bimolecular are marked with magnetic particles and are subsequently recognized by biomolecular probes immobilized at the surface of the chip over sensing sites. The markers fringe fields are then detected by magnetic tunneling junctions (MTJs) [1].

The developed system consists of a compact, credit card dimension, portable hand-held microsystem for biomolecular recognition assays. The microsystem includes the magnetoresistive biochip, and all the electronics necessary to address and read the sensors and to implement temperature and fluid control.

\section{ARCHITECTURE}

The proposed architecture for the biochip platform is organized in two main modules (fig. 1): $i$ ) the Sensing and Processing Module (SPM) and ii) the Fluid Control and Communications Module (FCCM). The SPM integrates the biochip and provides the circuits that directly interact with the array of biosensors (biochip), in order to individually address sensors, readout data from them, and to control the temperature in the different sub-areas of the biochip. The FCCM interfaces the platform with the external world by controlling the fluid carrying the magnetically tagged biomolecules and by providing wireless communication with a handheld analyzer based on a Personal Digital Assistant (PDA). All this is achieved using with only $100 \mathrm{~mA}$ of power consumption. The handheld device acts as the master of the system, giving the geneticist/biologist the biological measures.

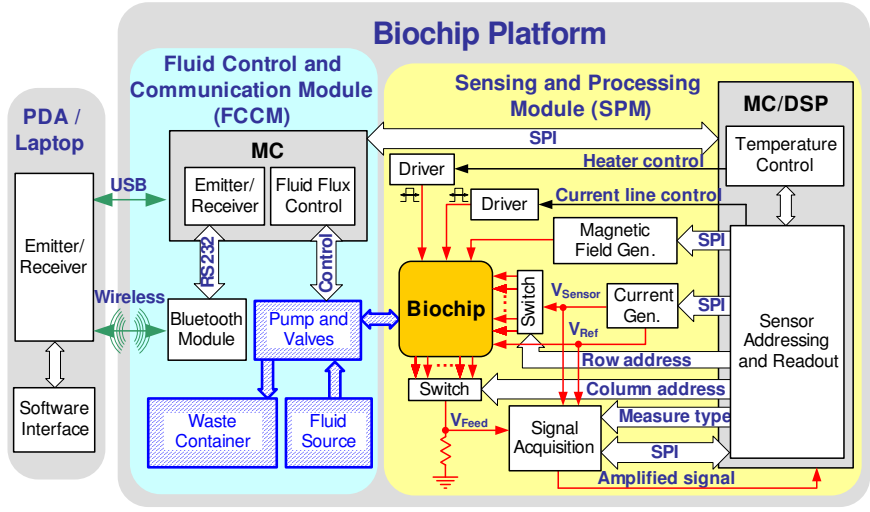

Fig. 1. Full diagram of the microsystem.

\section{A. Reading and controlling circuits}

The core of the system is a 16-bit integrated microcontroller (MicroController/Digital Signal Processor (MC/DSP)), the Microchip dsPIC 30F6014. This device has a performance up to 30 Million Instructions Per Second (MIPS) and an extended instruction set for digital signal processing. A Static Random Access Memory (SRAM) memory is also included in the SPM providing an additional memory space that can be used to store the acquired samples or the calibration data extracted from the biochip. The MC/DSP communicates with a microcontroller in the auxiliary board trough the Serial Peripheral Interface (SPI), and is programmed to perform a set of pre-defined tasks according to the commands received from the PDA. This MC/DSP addresses and reads the data from the array of magnetoresistive sensors provided by the biochip, and measures and controls the temperature by using those same devices. 


\section{SENSING SITES}

The schematic diagram of the biochip is presented in fig. 2. It is composed of a number of sensing sites, formed by a MTJ and a diode. The sites are arranged in a array and accessed through line and column wires that are selected through multiplexers outside the chip. The diode has two functions: to act as a commutator that prevents sites, other than the selected one, from being accessed (through loops represented as a dotted blue line in the figure), and to serve as a temperature sensor for biological reactions that take place on the chip. A typical set of diodes and magnetic tunnel junctions

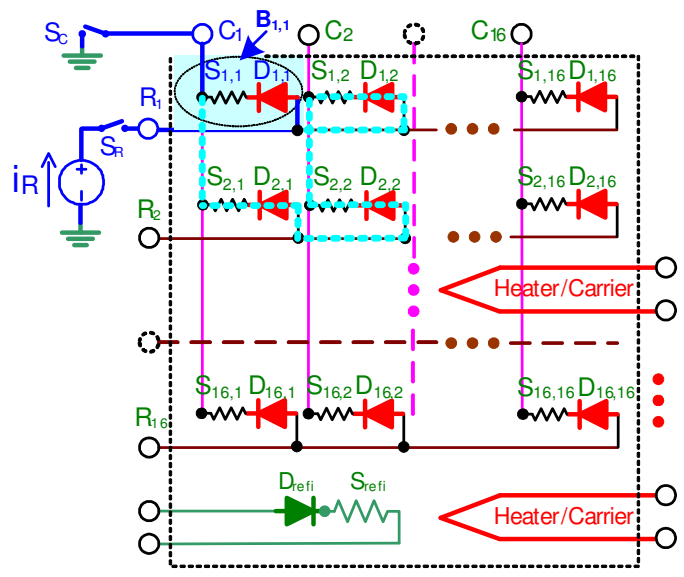

Fig. 2. Magnetoresistive Bio-chip schematic.

used in the biochip were characterized in [6].

\section{EXCITATION AND ACQUISITION}

The MTJs resistance varies with the applied magnetic field. In order to measure the resistance variation, some techniques must be applied. There are two obvious ways: i) to apply a constant voltage through a known resistor and measure the resulting voltage at the sensor site; ii) to apply a constant current at the site and measure the resulting voltage.

\section{A. Current or Voltage}

The two modes are in fact equivalent since the voltage source and the resistor can be made into a current source through the Norton theorem, but some differences may result in the dynamics of the system because of the different output impedances. The main components affecting the reading bandwidth at the sensor site are the diode capacitance, and the resistance seen by this capacitor. This is given by the parallel of the diode incremental resistance to the series of the signal source and sensor resistance. If the source resistance is larger, as in a current source, the bandwidth will be lower. However, in our system, the resistance seen by the sensor will be mostly dictated by the incremental resistance of the diode, causing the bandwidth of the system to be the same for voltage or current excitation. The use of a current source allows a simple calculation of the sensor resistor value, so this was the selected method.

\section{B. AC or DC measures}

Applying a current through the sensing site and measuring the voltage signal, will result in a signal that combines the voltage drop across the diode and across the MTJ. In order to extract the diode signal, one can simply subtract the signal measured before the insertion of the particle solution at the sensor. This was the procedure used in fig. 5. The result is a signal that is proportional to the concentration of particles in the solution.

However this signal is a small signal, that is absorbed in a large signal. A typical value for this may be about one hundred of a $\mu \mathrm{V}$, as shown in fig. 5. This was measured with a MTJ with a resistance of $10 \mathrm{k} \Omega$ and a $5 \mu \mathrm{A}$ DC current, so the signal level was $50 \mathrm{mV}$. This signal level requires the $\mathrm{AD}$ converter to have a large dynamic range. This problem can be reduced if the applied external magnetic field has a sinusoidal component. This alternating magnetic field will produce a corresponding variation on the resistance value. When this is multiplied by the values of the current source it will result in an AC signal, with the same frequency as the one used for generating the magnetic field. This signal can then be separated from the DC voltage through a high pass filter, and then amplified.

There is a trade off in selecting what type of measurement is done. One may choose to use a high dynamic range $\mathrm{AD}$ converter and no amplifier; an externally applied DC magnetic field, even with a permanent magnet; or use a lower dynamic range $\mathrm{AD}$ and an amplifier with an external $\mathrm{AC}$ field. The use of an AC external field also implies the use of a DAC, to drive the current into an external solenoid, which adds to the cost of the system and to the power consumption, but does not require the magnet. Another problem is the transitory response of the high pass filter. Since the working frequency is rather low, the filter has a high time constant. This will create a transitory response when ever there is a switch between sensors. If the time constant is too high it will limit the measuring rate of the system.

\section{Using a reference sensor}

In order to minimize the dynamic range required by the $\mathrm{AD}$ converter it is desirable to set the amplifier to the maximum gain, but without reaching saturation. Although the use of AC measurements improves this, the useful signal is still buried in a higher valued signal, because the MTJ is sensible to the full alternating $\mathrm{AC}$ field, and not only to the field created by the particles, which is usually smaller. This will be specially true when the junction is driven close to the magnetic saturation.

The problem can be further reduced if a reference sensor is used and the difference between the reference sensor and the measured sensor amplified. The measurement sensor can be selected through the multiplexers while the reference should be directly accessible. Due to the variation of system parameters from junction to junction this may not the best calibration technique, but it is useful for increasing the gain allowed to the amplifier. 


\section{AC current and DC field}

Another reason to switch to AC measurements is the noise levels, namely the low frequency noise, present in sensor and in the electronics. It can be seen in fig. 3 that the measured noise levels are higher at lower frequencies. This does not necessarily imply the use of an AC magnetic field. A DC field can be used when the sensing site is excited by the superposition of a bias DC current and a smaller AC signal. Because of non-linear behavior of the diode, a harmonic of the excitation signal will be present, but this can be easily be filtered out by the use of a moving average filter. Also, it is no longer possible to easily separate the MTJ resistance variation from its fixed value as in pure $\mathrm{AC}$ measures, so the signal cannot be greatly amplified, and a high-resolution AD is required.

\section{NOISE}

Measurements of noise levels in the board were made, for the case of a sinusoidal current excitation signal (AC mode). The sampling frequency was of $480 \mathrm{~Hz}$. The signal was a $30 \mathrm{~Hz}, 5 \mu \mathrm{A}$ current, injected through a $10 \mathrm{k} \Omega$ resistance.

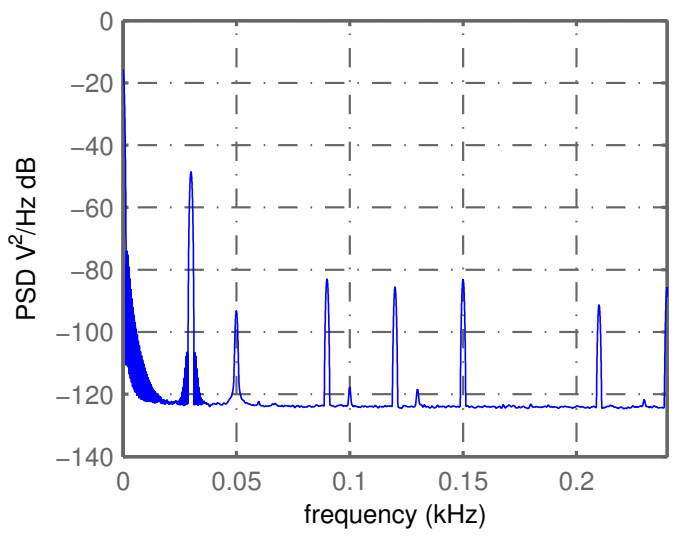

Fig. 3. Noise power spectral density at the sensor measured by the AD converter. A window of $0.47 \mathrm{~Hz}$ was used.

The noise power spectral density (PSD) can be seen in fig. 3. The noise is mostly composed of four components: Harmonics of the $30 \mathrm{~Hz}$ frequency, quantization noise from the DA converter; $50 \mathrm{~Hz}$ power line frequency noise; low frequency noise; and white noise. The total noise level is about $0.1 \mathrm{mV}_{\mathrm{RMS}}$, due to $\mathrm{DA}$ quantization. The $50 \mathrm{~Hz}$ power line noise and low frequency noise amount to $37 \mu \mathrm{V}_{\mathrm{RMS}}$ and the resulting noise is about $3.7 \mu \mathrm{V}_{\mathrm{RMS}}$. Further filtering with a $3.3 \mathrm{~s}$ length band-pass filter, reduces the noise to about $0.8 \mu \mathrm{V}_{\mathrm{RMS}}$. Techniques to eliminate $\mathrm{DA}$ quantization noise (signal harmonics) and the $50 \mathrm{~Hz}$ power line interference signal are shown in a later section. The white noise floor is at about $-120 \mathrm{~dB} \mathrm{~V} / \sqrt{\mathrm{Hz}}$.

Measurements of noise levels using a magnetic excitation signal and an MTJ were also made. The Root of the Power Spectral Density (RPSD) of the signals measured at the MTJ are presented in figure 4 . The signal was generated by an electromagnet excited by a sinusoidal magnetic signal (AC mode) with an amplitude of $25 \mathrm{Oe}$ and $31 \mathrm{~Hz}$ frequency. The sampling frequency was of $496 \mathrm{~Hz}$. A $2.5 \mu \mathrm{A}$ current was injected through the MTJ.

The resulting signal measured by the A/D converter (after the amplifier that was set to a gain of 191) had an amplitude of $0.025 \mathrm{~V}$ at the $31 \mathrm{~Hz}$ frequency. A change of the magnetic signal amplitude to 24.9 Oe resulted in a change in the amplitude of the signal measured by the A/D converted to $0.011 \mathrm{~V}$. Note that this implies that there isn't a linear relation between the applied field and the measured signal. This is due to the hysteresis curve of the junction. This values for the signals results in a sensitivity of $0.13 \mathrm{~V} / \mathrm{Oe}$.

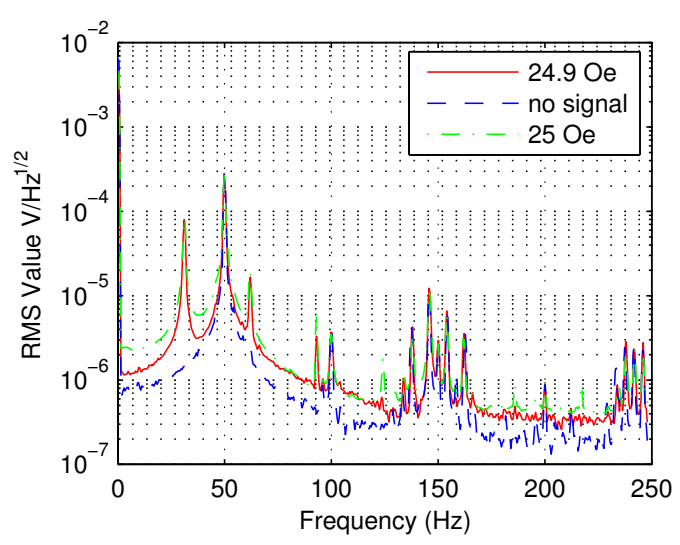

Fig. 4. Root power spectral density of the voltage signal at the MTJ measured by the A/D converter for a magnetic excitation signal. The magnetic field was of $25 \mathrm{Oe}, 24.9 \mathrm{Oe}$ and no signal. A window of $0.49 \mathrm{~Hz}$ was used.

\section{SignAl PROCESSING}

In the system there are two main tasks for the signal processor embedded in the board: the generation of the excitation signal and the recovery of the biological signal. Some of the signal processing techniques rely on previously obtained [3], [7] models for the MTJ and diode. For AC measurements, the chosen excitation signal was sinusoidal, while for DC a constant current was used. The sampling ratio was chosen to be a constant multiple of the sinusoidal signal. This allowed the signal to be generated very simply through a lookup table.

\section{A. Noise Filtering}

Assuming AC excitation, either with an AC field or an $\mathrm{AC}$ current, the amplitude of the voltage signal at the sensing site must be determined. The signal is assumed to be corrupted by white noise, with standard deviation $\sigma_{v}$, and the goal is to estimate the amplitude, $A$, of the signal. This can be done by calculating the DFT, and determine the amplitude at the excitation frequency. However, it is not required to calculate the full DFT, only its amplitude at the given frequency. This 


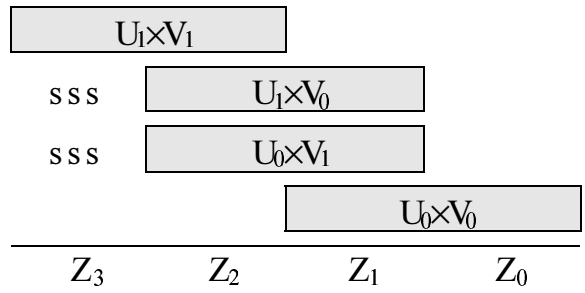

TABLE I

Simple 32Bits MULTIPLICATION.

can be calculated by,

$$
\begin{aligned}
A_{\text {DFT_real/imag }} & =\frac{\sum_{n=n_{0}}^{n_{0}+N-1} 2 y[n] \cos / \sin \left(\omega_{0} n\right)}{N} \\
A_{\text {DFT }} & =\sqrt{A_{\text {DFT_real }}^{2}+A_{\text {DFT_imag }}^{2}}
\end{aligned}
$$

This is an unbiased estimator of the amplitude. Its standard deviation, for small values of the noise signal, is given by,

$$
\sigma_{\left(A_{\mathrm{DFT}} / A\right)}=\sqrt{\frac{2}{N} \sigma_{(v / A)}} .
$$

\section{B. DSP implementation}

In order to save memory the DFT amplitude calculation was implemented in real time in the microsystem, using DSP capabilities of the microcontroller. The algorithms were implemented using a mix of $\mathrm{C}$ and assembly language. In order to use the full 24 bits of dynamic range of the AD converter the multiply and accumulate operations were implemented in double precision arithmetic, using 32 bits signed integers(although the DSP is a 16bits processor with 16bits register and operations). The multiplication operation implemented takes only 29 instruction cycles and calculates $\operatorname{long}_{32}=\operatorname{round}\left(\operatorname{long}_{32} \times \operatorname{long}_{32}>>32\right)$. A single precision floating point operation takes 128 instruction cycles. Other possible implementations required as many as 1765 instruction cycles. The computational cost is very far from exhausting the computational power of the DSP but lower cycle count allows a reduction of the consumption of the board. The square root operation was implemented in single precision floating point (32 bits) using available $\mathrm{C}$ libraries.

The 32 bits multiplication was implemented using a simple multiply algorithm. Given a 32bits signed number $U$ it can be expressed as a signed and an unsigned 16 bits numbers, $U=$ $U_{1 \mid \text { signed }} 2^{16}+U_{0 \mid \text { unsigned }}$. Using the distributive property, the 32 bits multiplication can be implemented with four 16bits multiplications and some additions. Table I illustrates the algorithm (note that "sss" represents sign extension).

\section{EXPERIMENTS}

The microsystem was tested using a solution of $2.3 \times$ $10^{9}$ particles $/ \mathrm{ml}$ with $1.5 \mu \mathrm{m}$ diameter magnetic nanoparticles. An $5 \mu \mathrm{A}$ DC current was driven by the DAC through a $10 \mathrm{k} \Omega$ MTJ. The voltage signal was measured by an AD converter at a sample rate of $6 \mathrm{~Hz}$ after passing through a suitable antialiasing filter. The measurement time was about 8 minutes. The measured signal is presented in fig. 5, after the removal of a $47 \mathrm{mV}$ DC signal. The solution was dropped on the sensor after about 1000 samples and after about 1750 samples the sensor was washed with distilled water. The figure clearly shows a $150 \mu \mathrm{V}$ signal due to the presence of nanoparticles, demonstrating that the microsystem can be used for particle detection. The noise at the sensor could be further reduced by averaging. Also, if the current level was increased to $50 \mu \mathrm{A}$, the signal level could be increased to $300 \mu \mathrm{V}$ but this would lead to higher noise levels and lower signal to noise ratio.

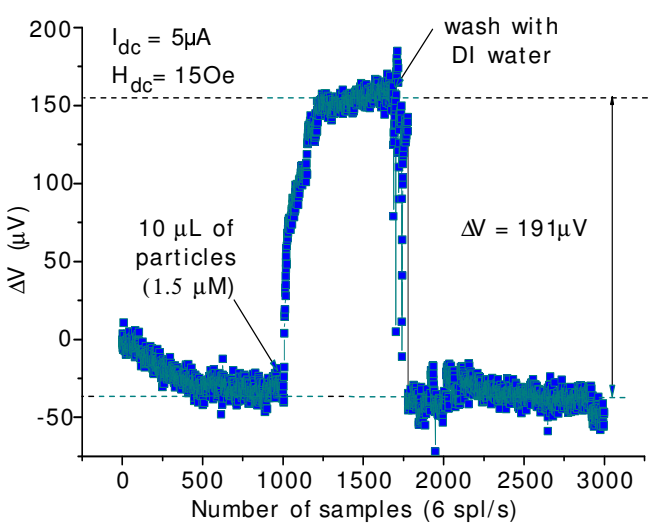

Fig. 5. Time variation of the measured signal for evaluation of particle detection capabilities.

\section{CONCLUSION}

A recently developed handheld biochip-based Microsystems was presented. Different types of measures were compared. Filtering techniques based on noise signals presented in the system were presented. Finally, the viability of the system was demonstrated by testing it in a real bio signal detection experiment with good results.

\section{REFERENCES}

[1] W. Shen, X. Liu, D. Mazumdar, and G. Xiao, "In situ detection of single micron-sized magnetic beads using magnetic tunnel junction sensors," Appl. Phys. Lett., vol. 86, no. 25, p. 253901, June 2005.

[2] "The chipping forecast, suplement," Nature Genet., vol. 21, no. 1, p. 60, January 1999.

[3] J. Germano et al., "Microsystem for biological analysis based on magnetoresistive sensing," in XVIII International Measurement Confederation (IMEKO) World Congress, Rio de Janeiro, Brazil, 2006.

[4] M. Johnson, Magnetoelectronics. Academic Press, 2004, ch. 7, pp. 331274.

[5] D. L. Graham, H. A. Ferreira, and P. P. Freitas, "Magnetoresistive-based biosensors and biochips," Trends Biotechnol, vol. 22, no. 9, pp. 455-462, September 2004.

[6] T. M. Almeida et al., "Characterisation and modelling of a magnetic biosensor,' in Instrumentation and Measurement Technology Conference 2006, Sorrento, Italy, 2006.

[7] — "Magnetoresistive biosensor modelling for biomolecular recognition," in XVIII International Measurement Confederation World Congress, Rio de Janeiro, Brazil, 2006. 